\title{
KPNA3 variation is associated with schizophrenia, major depression, opiate dependence and alcohol dependence
}

\author{
Charles P. Morris ${ }^{\mathrm{a}}$, Bernhard T. Baune ${ }^{\mathrm{c}}$, Katharina Domschke ${ }^{\mathrm{d}}$, Volker Arolt ${ }^{\mathrm{d}}$, Christopher D. Swagell ${ }^{\mathrm{a}}$, \\ Ian P. Hughes ${ }^{\mathrm{a}}$, Bruce R. Lawford ${ }^{\mathrm{b}}$, Ross McD Young ${ }^{\mathrm{a}}$ and Joanne Voisey ${ }^{\mathrm{a}, *}$ \\ ${ }^{\mathrm{a}}$ Institute of Health and Biomedical Innovation, Queensland University of Technology, Brisbane, Queensland, \\ Australia \\ ${ }^{\mathrm{b}}$ Division of Mental Health, Royal Brisbane and Women's Hospital, Brisbane, Queensland, Australia \\ ${ }^{\mathrm{c}}$ Department of Psychiatry, School of Medicine, University of Adelaide, Adelaide, South Australia, Australia \\ ${ }^{\mathrm{d}}$ Department of Psychiatry, University of Muenster, Muenster, Germany
}

\begin{abstract}
KPNA3 is a gene that has been linked to schizophrenia susceptibility. In this study we investigated the possible association between KPNA3 variation and schizophrenia. To investigate a wider role of KPNA3 across psychiatric disorders we also analysed major depression, PTSD, nicotine dependent, alcohol dependent and opiate dependent cohorts. Using a haplotype block-based gene-tagging approach we genotyped six KPNA3 single nucleotide polymorphisms (SNPs) in 157 schizophrenia patients, 121 post-traumatic stress disorder patients, 120 opiate dependent patients, 231 alcohol dependent patients, 147 nicotine dependent patients and 266 major depression patients. One SNP rs2273816 was found to be significantly associated with schizophrenia, opiate dependence and alcohol dependence at the genotype and allele level. Major depression was also associated with rs2273816 but only at the allele level. Our study suggests that KPNA3 may contribute to the genetic susceptibility to schizophrenia as well as other psychiatric disorders.
\end{abstract}

Keywords: KPNA3, genetic, schizophrenia, polymorphism, psychiatric disorder, cross-disorder

\section{Introduction}

Karyopherin alpha-3 (KPNA3) encodes a 521 amino acid protein that is highly homologous to nuclear transport proteins including xenopus importin, yeast importin alpha 1 and human importin alpha 1 . The protein contains an $\mathrm{N}$-terminal importin-beta-binding domain, 8 armadillo repeats and a C-terminal acidic region which are common to importin-alphas [16]. It is likely that karyopherins are responsible for the trans-

* Corresponding author: Joanne Voisey, Institute of Health and Biomedical Innovation, Queensland University of Technology, Brisbane, Queensland, Australia. Tel.: +61 7 31386261; Fax: +61 7 31386030; E-mail: j.voisey@qut.edu.au. port of macromolecules (containing nuclear import or export signals) between the nucleus and cytoplasm [24].

The KPNA3 gene was mapped to $13 \mathrm{q} 14.3$ by fluorescence in situ hybridization analysis [32]. Linkage studies have revealed this region to be of interest in schizophrenia susceptibility [3,9,21]. KPNA3 has been showed to be associated with schizophrenia in a British [37] and Chinese population [41]. The British study analysed 124 family trios consisting of mother, father and affected offspring and association with schizophrenia was found with three $K P$ NA3 polymorphisms including rs3736830, rs2181185 and rs626716 [37]. In the Chinese study investigating two KPNA3 polymorphisms, 238 family trios consisting of mother, father and affected offspring showed association with schizophrenia [41] and rs3782929 but 
not rs3736830. In a Bulgarian population, association between KPNA3 and schizophrenia was not found [6].

As well as being associated with schizophrenia, KPNA3 may also be associated with other psychiatric conditions as chromosome 13 overlaps a few psychiatric disorders. A linkage scan has identified chromosome 13 of interest for alcohol and illicit drug dependence but this region does not overlap KPNA3 [2]. Other studies show that chromosome $13 \mathrm{q} 14$ is a susceptibility locus for mood disorder and bipolar disorder [19,23]. To date KPNA3 has not been analysed for association with other psychiatric disorders. Other genes such as DRD 2 and DTNBP1 have been shown to be associated with a number of psychiatric conditions. The DRD2 polymorphism rs6277 was first found to be associated with schizophrenia [18] but has since found to be associated with working memory [40], alcohol dependence [28], impulsivity [38] and post traumatic stress disorder (PTSD) [34]. We have also established that a polymorphism in DTNBP1 (rs9370822) is common to a range of psychiatric conditions including schizophrenia [36], psychotic depression [12] and PTSD, nicotine dependence and opiate dependence [35].

Schizophrenia is a complex multifactorial disorder, showing polygenic inheritance. Therefore to begin to understand the complexity of schizophrenia many polymorphisms in multiple genes need to be identified for association with disease and results validated. We undertook our study to replicate the previous KPNA3 associations with schizophrenia in an Australian population and identify novel SNP associations. To identify any common molecular mechanism across psychiatric disorders, we genotyped KPNA3 polymorphisms that were found significantly associated with schizophrenia in other cohorts including major depression, posttraumatic stress disorder (PTSD), alcohol dependent, nicotine dependent and opiate dependent groups. This is the first study that has undertaken an analysis of KPNA3 across a range of psychiatric disorders.

\section{Materials and methods}

\subsection{Participants}

\subsubsection{Controls}

The control group consisted of 237 unrelated Caucasians (98 female and 139 male) with a mean age of 36.8 years (s.d. \pm 12.8 years). The control group consisted of volunteers from the general public, hospital nursing and medical staff, and university staff and students. Formal screening for psychological disorders was not undertaken in the control population. As such the controls represent an unselected control group. Controls were recruited in the Brisbane region (a city of approximately 2 million inhabitants on the East Coast of Australia) and all were of Caucasian descent.

\subsubsection{Schizophrenia}

The schizophrenia group consisted of 157 Caucasian patients. Subjects were aged between 18 and 65 years. Diagnostic and Statistical Manual of Mental DisordersIV (DSM-IV) diagnosis of schizophrenia was confirmed by at least two independent psychiatrists. These patients had never been diagnosed with other psychiatric disorders, including schizoaffective disorder, major depressive episode with psychotic features, or bipolar disorder. There were 23 females and 134 males in the group diagnosed with schizophrenia with a mean age of 36.2 years (s.d. \pm 12.0 years). The mean age of patients at first diagnosis (onset of psychotic symptoms) was 23.2 years (s.d. \pm 7.3 years). They were being treated at the Royal Brisbane and Women's Hospital, The Park Psychiatric Unit, and the Valley Community Psychiatric Centre. The clinical sample was composed of 65 inpatients and 92 outpatients. All patients were administered the Positive and Negative Symptom Scale (PANSS) [27] to assess severity of psychotic features. The PANSS total mean score was 45.13, SD. 13.69. This clinical sample was a group who had lived with schizophrenia for an average of 13 years since diagnosis and continued to experience positive and negative symptoms despite treatment with antipsychotic medication. Mean length of illness was 13.3 years, SD 10.6. In addition the schizophrenia group contained a high proportion of individuals with a relatively severe history and/or a familial risk for psychosis.

\subsubsection{Opiate dependence}

A total of 120 unrelated Caucasian participants (50 female and 70 male) diagnosed as opiate dependent were recruited for this study, all of whom were being assessed for naltrexone treatment as outpatients of the Hospital Alcohol and Drug Services Unit of the Royal Brisbane and Women's Hospital. Participants had a mean age of 30.0 years of age (s.d. \pm 7.9 years). Approximately half of the participants were being managed on methadone prior to detoxification $(47.2 \%)$ while the other half were on heroin $(52.8 \%)$. Those on methadone had a mean dose of 48.1 milligrams (s.d. 30.5 ), with a range between 10-165 milligrams. The mean age of onset of heroin use was 22.4 years of age 
(s.d. 5.14), with a range between $15-43$ years. Mean number of participant-reported detoxifications prior to this occasion was 3.5 (s.d. 3.3), with a range between 0-16 detoxifications. Cannabis was the most commonly concurrently used illicit substance reported by participants prior to treatment $(52.5 \%$ reported use), followed by amphetamines ( $14.9 \%$ reported use). Individuals were excluded from the study if they suffered from psychosis, depression or PTSD. Individuals were not excluded from the study if they were smokers and approximately $90 \%$ were smokers.

\subsubsection{PTSD}

A total of 121 unrelated male Caucasian patients diagnosed with PTSD using Diagnostic and Statistical Manual of Mental Disorders-IV (DSM-IV) criteria were recruited through the Greenslopes Private Hospital for the study. Participants had a mean age of 52 years of age (s.d. \pm 6.2 ). All subjects were Vietnam combat veterans who had served in the Australian Armed Forces. None were being treated with psychotropic medications. Patients were excluded from the study if they had a diagnosis of psychosis, bipolar disorder, obsessive compulsive disorder, or organic brain syndrome including dementia. Patients were excluded from the study if that had opiate dependence. All subjects had sufficient comprehension of English and could understand the administered questionnaires. Patients were assessed for PTSD by a consultant psychiatrist or a senior psychiatric registrar using DSM-IV criteria. Furthermore, every patient exceeded the clinical cut-off score of 94 on the Mississippi Scale for combat-related PTSD [15]. Patient clinical history and demographic data including ethnic background were also obtained. After the procedure had been fully explained, all participants provided written informed consent. They were able to terminate their involvement at any time during the interview without prejudice. Institutional ethics approval was obtained from the Greenslopes Private Hospital.

\subsubsection{Alcohol dependence}

A total of 231 unrelated Caucasian (74 female and 157 male) alcohol dependent subjects were recruited from large public hospitals in Brisbane, Australia. The mean age of the group was 40.74 years (s.d. \pm 10.3 years). All subjects were assessed against a checklist of specific criteria by a clinical psychologist experienced in drug and alcohol dependence and met DSMIV (Diagnostic and Statistical Manual of Mental Disorders IV) criteria of alcohol dependence disorder. All were inpatients and represented a spectrum of severity with a significant proportion $(n=65)$ of these patients being diagnosed with two or more alcohol related medical conditions such as pancreatitis, cirrhosis, hepatitis or peripheral neuropathy. Alcohol dependent patients were excluded from the study if they had dementia, delirium, psychosis, or any other condition that would affect their ability to provide informed consent. A large proportion $(81 \%)$ of the alcohol dependent cohort was also smokers.

\subsubsection{Nicotine dependence}

A total of 147 (68 male, 79 female) unrelated Caucasians with a mean age of 43.3 (s.d. \pm 11.1 years) were recruited from large public hospitals in Brisbane, Australia. Participants were 18 years of age or older and had smoked for at least three years and were generally healthy despite currently smoking 15 cigarettes or more per day. Patients with other comorbid substance abuse disorders including alcohol and opiate dependence were excluded from the study. One hundred and thirty nine of the participants were administered the Fagerstrom test for Nicotine Dependence [14].

\subsubsection{Major depression}

A sample of 266 (113 male, 153 female) unrelated Caucasian patients with a current major depression (mean age $49.7 \pm 15.3$ years) admitted for in-patient treatment were recruited at the Department of Psychiatry, University of Muenster, Germany. Patients with schizoaffective disorders, bipolar disorder or comorbid substance abuse disorders, mental retardation, pregnancy and neurological, neurodegenerative disorders or other clinically unstable medical illnesses impairing psychiatric evaluation were not included in this analysis [4,11].

Ethics approval was obtained from all institutions involved and each participant gave written informed consent. The study conforms with The Code of Ethics of the World Medical Association (Declaration of Helsinki), printed in the British Medical Journal (18 July 1964).

\subsection{Selection of SNPs}

Using the International HapMap project phase II (International HapMap, 2003), five tag-SNPs in KPNA3 with a minor allele frequency cut-off of 0.15 were selected. Tag-SNPs were identified using the pairwise option of Tagger with a threshold of $\mathrm{r}^{2}>0.8$. An additional non-synonymous SNP was also chosen 
Table 1

Allele Association of 6 KPNA3 SNPs with schizophrenia

\begin{tabular}{llccccc}
\hline SNP ID & Position* $^{*}$ & SNP details & $\chi^{2}$ & p-value & Odds ratio & 95\% CI \\
\hline rs1407439 & 49181116 & Intron 7 A/G & 1.112 & 0.292 & 1.20 & $0.85-1.72$ \\
rs2273816 & 49192057 & Intron 5 A/G & 8.924 & 0.003 & 1.96 & $1.22-3.15$ \\
rs10450845 & 49205117 & Asp61 Asn & \multicolumn{4}{c}{ Not polymorphic in this study } \\
rs9562919 & 49221809 & Intron 1 A/T & 0.748 & 0.387 & 1.14 & $0.84-1.54$ \\
rs1923730 & 49229993 & Intron 1 A/G & 0.210 & 0.647 & 1.08 & $0.77-1.49$ \\
rs2236354 & 49263967 & Intron 1 C/T & 0.275 & 0.600 & 1.09 & $0.78-1.52$ \\
\hline
\end{tabular}

* Nucleotide position on the chromosome 13 reference sequence.

$p$-value determined by Pearson's chi-squared test.

Table 2

Allele Association of KPNA3 SNP rs2273816 with multiple psychiatric conditions

\begin{tabular}{lcccc}
\hline Sample set & $\chi^{2}$ & p-value & Odds ratio & 95\% CI \\
\hline Schizophrenia & 8.924 & 0.003 & 1.96 & $1.22-3.15$ \\
PTSD & 2.297 & 0.130 & 1.47 & $0.86-2.48$ \\
Nicotine dependence & 2.755 & 0.097 & 1.49 & $0.90-2.47$ \\
Opiate dependence & 6.036 & 0.014 & 1.82 & $1.09-3.03$ \\
Alcohol dependence & 7.916 & 0.005 & 1.81 & $1.17-2.84$ \\
Major Depression & 5.556 & 0.018 & 1.63 & $1.07-2.52$ \\
\hline${ }^{*} p$-value determined by Pearson's chi-squared test. & &
\end{tabular}

for analysis. Using the Stampa algorithm it was calculated that $94.14 \%$ of the variation in the KNPA3 gene was captured using the five tag SNPs (rs1407439, rs2273816, rs9562919, rs1923730, rs2236354) and one non-synonymous SNP (rs10450845) [13].

\subsection{Genotyping}

Oragene kits were used to extract DNA from saliva samples. Samples were genotyped using a homogeneous MassEXTEND (hME) Sequenom assay performed by the Australian Genome Research Facility. The hME assay is based on the annealing of an oligonucleotide primer (hME primer) adjacent to the SNP of interest. The addition of a DNA polymerase along with a mixture of terminator nucleotides allows extension of the hME primer through the polymorphic site and generates allele-specific extension products, each having a unique molecular mass. The resultant masses of the extension products are then analysed by matrixassisted laser desorption/ionization time-of-flight mass spectrometry (MALDI-TOF MS) and a genotype is assigned in real time.

\subsection{Statistical analysis}

A Fisher's exact test was performed to identify statistical associations between genotype and disease status. A Pearson's chi-squared test was performed to identify statistical associations between allele and disease status. Odds ratios (OR) were also calculated. Tests were performed on both genotype and allele data. Statistical tests were performed using the COMPARE2 program from the WinPepi suite of epidemiology programs [1] and SPSS version 16. Hardy-Weinberg equilibrium (HWE) was computed using Utility Programs for Analysis of Genetic Linkage [26]. Correction for multiple testing was conducted using the false detection rate (FDR) method [5]. Haplotypes were generated and analysed for linkage disequilibrium (LD) measures (D' and $\mathrm{r}^{2}$ ) using JLIN [10].

\section{Results}

\subsection{Schizophrenia genotyping}

A comprehensive genotype analysis of KPNA3 and its association with schizophrenia was undertaken using a haplotype block-based gene-tagging approach. A total of five tag-SNPs and one synonymous SNP that span from the 3'-end to the 5'-end of the gene were genotyped in a sample of 157 schizophrenia patients and 237 controls. One SNP rs2273816 was found to be significantly ( $p=0.003$ ) associated with schizophrenia at the allele level (Table 1). It was also significant at the genotype level (Table 3 ) and survived correction for multiple testing (FDR corrected $\alpha$-value was 0.01).

The attributable fraction of the rs 2273816 polymorphism was calculated using genotypes counting the presence of the associated allele as a risk factor. The attributable fraction, which is the proportion of cases 
Table 3

Genotype Association of KPNA3 SNP rs2273816

\begin{tabular}{|c|c|c|c|c|}
\hline \multirow[t]{2}{*}{ Sample set } & \multicolumn{3}{|c|}{ Genotype counts } & \multirow[t]{2}{*}{ p-value* } \\
\hline & GG (\%) & $\mathrm{GA}(\%)$ & $\mathrm{AA}(\%)$ & \\
\hline control & $180(83.33)$ & $32(14.82)$ & $4(1.85)$ & 0.005 \\
\hline Schizophrenia & $102(69.39)$ & $41(27.89)$ & $4(2.72)$ & $0.004 \dagger$ \\
\hline Odds ratio & 1 & 2.261 & 1.765 & \\
\hline$p$-value & & 0.004 & 0.863 & \\
\hline PTSD & $89(74.79)$ & $29(24.37)$ & $1(0.84)$ & 0.074 \\
\hline Odds ratio & 1 & 1.833 & 0.506 & $0.138 \dagger$ \\
\hline$p$-value & & 0.072 & 1.000 & \\
\hline Nicotine dependence & $108(77.14)$ & $27(19.29)$ & $5(3.57)$ & 0.307 \\
\hline Odds ratio & 1 & 1.406 & 2.083 & $0.121 \dagger$ \\
\hline$p$-value & & 0.478 & 0.561 & \\
\hline Opiate dependence & $81(70.43)$ & $32(27.83)$ & $2(1.74)$ & 0.015 \\
\hline Odds ratio & 1 & 2.222 & 1.111 & $0.017 \dagger$ \\
\hline$p$-value & & 0.010 & 1.000 & \\
\hline Alcohol dependence & $148(71.15)$ & $55(26.44)$ & $5(2.41)$ & 0.010 \\
\hline Odds ratio & 1 & 2.090 & 1.520 & $0.006 \dagger$ \\
\hline$p$-value & & 0.005 & 1.000 & \\
\hline Major Depression & $191(74.61)$ & $57(22.27)$ & $8(3.12)$ & 0.066 \\
\hline Odds ratio & 1 & 1.679 & 1.885 & $0.025 \dagger$ \\
\hline$p$-value & & 0.063 & 0.590 & \\
\hline
\end{tabular}

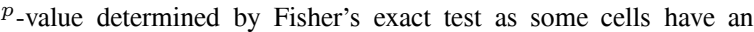
expected frequency $<5$.

$\dagger p$-value determined using the extended Mantel-Haenszel test for trend.

in the study population that would not have occurred had the risk factor (risk allele) not have been present, is a useful measure to quantify the impact of the allele on the development of schizophrenia [17]. The analysis revealed that $15.56 \%(95 \% \mathrm{CI}=5.29-24.71 \%)$ of the susceptibility to schizophrenia could be attributed to having the GA genotype of the rs 2273816 polymorphism.

Haplotype analysis was completed and the results revealed no improvement in association with any haplotype combinations compared to the rs2273816 SNP analysed in isolation. The best haplotype combination was with rs2273816 and rs9562919 $(p=0.026)$. Furthermore, the rs2273816 SNP showed low LD with all other SNPs while the other SNPs were in very high disequilibrium.

Genotype frequencies indicated that all polymorphisms were in HWE in both schizophrenia and control samples.

\subsection{Major depression, PTSD, nicotine dependence, opiate dependence and alcohol dependence genotyping}

To investigate the role of rs2273816 in a range of psychiatric conditions this SNP was genotyped in 266 unipolar major depression samples, 121 PTSD samples, 154 nicotine dependent samples, 120 opiate de- pendent samples and 231 alcohol dependent samples. The rs2273816 SNP was found to be associated with alcohol dependence, opiate dependence and major depression at the allele level but not PTSD or nicotine dependence (Table 2). At the genotype level rs2273816 was still associated with alcohol and opiate dependence but not major depression unless it was tested using the Mantel-Haenszel test for trend (Table 3). Individuals with alcohol or opiate dependence were more than twice as likely to carry the GA genotype compared to the control group. Genotype frequencies indicated that rs2273816 was in HWE for all samples including controls, major depression, PTSD, nicotine dependent, opiate dependent and alcohol dependent samples.

For the two groups, nicotine dependence and PTSD that did not show association, we did not have sufficient power $(<0.80)$ to detect association with our sample sizes.

\section{Discussion}

Schizophrenia is not a single disorder of uniform phenotype; it exists as a clinically variable spectrum of disease with some features that overlap other recognised psychiatric disorders. We therefore postulated that genes involved in schizophrenia like KPNA3 might also play a role in other disorders. This is the first study to investigate an association between the KPNA3 gene and a range of psychiatric conditions. KPNA3 has not been intensively investigated for a cross-disorder role in various psychiatric diseases even though it is situated in a region highly associated with schizophrenia risk. Only two studies have shown that KPNA3 is associated with schizophrenia $[37,41]$. We sought to replicate these associations with KPNA3 and schizophrenia as well as investigate the role of KPNA3 in various psychiatric conditions in order to search for possible common pathways across psychiatric disorders. In our study we found the KPNA3 SNP rs2273816 to be significantly associated with schizophrenia, opiate dependence and alcohol dependence at the genotype level. Major depression was also associated with rs2273816 but only at the allele level.

A British study also found rs22731816 to be associated with schizophrenia but only with rs3736830 as a haplotype [37]. They also found rs 3736830 to be associated with disease on its own. In our study we did not genotype the rs3736830 polymorphism. Like our study, their sample size was also relatively small and this association needs to be replicated in a larger sample 
size and possibly different ethnic groups. A Chinese study failed to find association with rs 3736830 but this maybe because of ethnicity [41]. Genome Wide Association Studies (GWAS) have not found KPNA3 to be associated with schizophrenia [29-31]. However, if KPNA3 confers small disease risk then it would be unlikely to be found using GWAS where the current $P$ value threshold is $5 \times 10^{-8}$ taking into account multiple testing $[7,25]$. Schizophrenia is a complex multifactorial disorder where genetic and environmental factors confer risk. The genetic component is likely the result from numerous polymorphisms in many genes that individually have a small effect. It is now believed that rare polymorphisms that account for a large percentage of schizophrenia heritability will not be captured using GWAS [33,39].

Interestingly, KPNA3 is also involved in the nuclear import of the influenza virus [22]. Although not conclusive, evidence does suggest that gestational exposure to influenza contributes to the etiology of schizophrenia $\{$ reviewed [8]\}. Therefore it is plausible that KPNA3 acts as a susceptibility gene for schizophrenia only after prenatal infection. Unfortunately we did not collect data on maternal exposure to infection. To answer this question, schizophrenia patients that have been exposed to an infection including influenza would be genotyped for KPNA3 genetic variants. It is unlikely that exposure to the influenza virus is involved in the etiology of alcohol and opiate dependence. However like schizophrenia, exposure to the influenza virus may contribute to the etiology of major depression although to date there has been no evidence to support this.

There are a number of possible limitations of this study. Population stratification can produce false positive associations where the underlying structure of the population results in genetic differences in the frequency of variants rather than differences due to the presence of disease susceptibility alleles. Every effort was made to ensure the uniformity of the population by selecting patients and controls of European descent however we cannot rule out population stratification and our results need to be confirmed independently in a Caucasian group as well as defined ethnic groups. The best research practice is for an experienced mental health professional to perform a structured interview (SCID) to generate patient diagnosis. A limitation of our study is that we did not perform a SCID except for the depression group. However, we did ensure all participants met DSM1V criteria and were diagnosed by more than one experienced mental health professional. The samples size of each psychiatric condition studied was rel- ative small and requires replication in larger independent patient groups. Association was not detected for the nicotine dependent and PTSD groups. The sample size was large enough to detect a strong genetic effect but to determine if these groups are also associated with KPNA3 variation with an OR less than 1.5 , a population size of more than 700 patients for each group would be required to give sufficient power. Our controls were not screened for a mental illness however, more significant associations are likely to be found using controls screened for a lack of mental illness.

This is a preliminary study and requires replication in a larger sample cohort with defined psychiatric diagnoses. Although many association studies often report conflicting results it is likely that many of these candidate associations independently have a minor effect on disease and may produce varied results depending on the severity of disease and the heterogeneity and ethnicity of the population. It is possible that KPNA3 has a minor effect on the susceptibility to schizophrenia, major depression and substance misuse through a common molecular pathway. It is not surprising that a common pathway across these psychiatric disorders may be involved since depression and substance misuse are often seen as comorbid conditions in patients with schizophrenia [20].

\section{Acknowledgements}

This work was financially supported by the Queensland State Government, the Nicol Foundation and the Institute of Health and Biomedical Innovation, QUT. JV is a Queensland Smart State Fellow.

\section{References}

[1] J. H. Abramson, Winpepi (pepi-for-windows): Computer programs for epidemiologists, Epidemiol Perspect Innov 1 (2004), 6.

[2] A. Agrawal, A. L. Hinrichs, G. Dunn, S. Bertelsen, D. M. Dick, S. F. Saccone, N. L. Saccone, R. A. Grucza, J. C. Wang, C. R. Cloninger, H. J. Edenberg, T. Foroud, V. Hesselbrock, J. Kramer, K. K. Bucholz, S. Kuperman, J. I. Nurnberger, Jr., B. Porjesz, M. A. Schuckit, A. M. Goate, L. J. Bierut, Linkage scan for quantitative traits identifies new regions of interest for substance dependence in the collaborative study on the genetics of alcoholism (coga) sample, Drug Alcohol Depend 93 (2008), 12-20.

[3] J. A. Badner, E. S. Gershon, Meta-analysis of whole-genome linkage scans of bipolar disorder and schizophrenia, Mol Psychiatry 7 (2002), 405-411. 
[4] B. T. Baune, C. Hohoff, L. S. Mortensen, J. Deckert, V. Arolt, K. Domschke, Serotonin transporter polymorphism (5-httlpr) association with melancholic depression: A female specific effect? Depress Anxiety 25 (2008), 920-925.

[5] Y. Benjamini, Y. Hochberg, Controlling the false discovery rate: A practical and powerful approach to multiple testing, Journal of the Royal Statistical Society, Series B 57 (1995), 289-300.

[6] E. T. Betcheva, T. Mushiroda, A. Takahashi, M. Kubo, S. K. Karachanak, I. T. Zaharieva, R. V. Vazharova, Dimova, II, V. K. Milanova, T. Tolev, G. Kirov, M. J. Owen, M. C. O'Donovan, N. Kamatani, Y. Nakamura, D. I. Toncheva, Casecontrol association study of 59 candidate genes reveals the drd2 snp rs6277 (c957t) as the only susceptibility factor for schizophrenia in the bulgarian population, J Hum Genet $\mathbf{5 4}$ (2009), 98-107.

[7] B. Bondy, Genetics in psychiatry: Are the promises met?, World J Biol Psychiatry 12 (2011), 81-88.

[8] A. S. Brown, E. J. Derkits, Prenatal infection and schizophrenia: A review of epidemiologic and translational studies, $\mathrm{Am}$ J Psychiatry 167 (2010), 261-280.

[9] N. J. Camp, S. L. Neuhausen, J. Tiobech, A. Polloi, H. Coon, M. Myles-Worsley, Genomewide multipoint linkage analysis of seven extended palauan pedigrees with schizophrenia, by a markov-chain monte carlo method, Am J Hum Genet 69 (2001), 1278-1289.

[10] K. W. Carter, P. A. McCaskie, L. J. Palmer, Jlin: A java based linkage disequilibrium plotter, BMC Bioinformatics 7 (2006), 60.

[11] K. Domschke, C. Hohoff, L. S. Mortensen, T. Roehrs, J. Deckert, V. Arolt, B. T. Baune, Monoamine oxidase a variant influences antidepressant treatment response in female patients with major depression, Prog Neuropsychopharmacol Biol Psychiatry 32 (2008), 224-228.

[12] K. Domschke, B. Lawford, R. Young, J. Voisey, C. P. Morris, T. Roehrs, C. Hohoff, E. Birosova, V. Arolt, B. T. Baune, Dysbindin (dtnbp1) - a role in psychotic depression?, J Psychiatr Res Epub ahead of print PMID:20951386 (2010).

[13] E. Halperin, G. Kimmel, R. Shamir, Tag snp selection in genotype data for maximizing snp prediction accuracy, Bioinformatics 21(Suppl 1) (2005), i195-203.

[14] T. F. Heatherton, L. T. Kozlowski, R. C. Frecker, K. O. Fagerstrom, The fagerstrom test for nicotine dependence: A revision of the fagerstrom tolerance questionnaire, $B r J$ Addict $\mathbf{8 6}$ (1991), 1119-1127.

[15] T. M. Keane, J. M. Caddell, K. L. Taylor, Mississippi scale for combat-related posttraumatic stress disorder: Three studies in reliability and validity, J Consult Clin Psychol 56 (1988), 85-90.

[16] M. Kohler, S. Ansieau, S. Prehn, A. Leutz, H. Haller, E. Hartmann, Cloning of two novel human importin-alpha subunits and analysis of the expression pattern of the importin-alpha protein family, FEBS Lett 417 (1997), 104-108.

[17] J. Lappalainen, H. R. Kranzler, R. Malison, L. H. Price, C. Van Dyck, R. A. Rosenheck, J. Cramer, S. Southwick, D. Charney, J. Krystal, J. Gelernter, A functional neuropeptide y leu7pro polymorphism associated with alcohol dependence in a large population sample from the united states, Arch Gen Psychiatry 59 (2002), 825-831.

[18] B. R. Lawford, R. M. Young, C. D. Swagell, M. Barnes, S. C. Burton, W. K. Ward, K. R. Heslop, S. Shadforth, A. van Daal, C. P. Morris, The $\mathrm{c} / \mathrm{c}$ genotype of the $\mathrm{c} 957 \mathrm{t}$ polymorphism of the dopamine $\mathrm{d} 2$ receptor is associated with schizophrenia, Schizophr Res 73 (2005), 31-37.
[19] D. F. Levinson, Meta-analysis in psychiatric genetics, Curr Psychiatry Rep 7 (2005), 143-151.

[20] D. A. Lewis, J. A. Lieberman, Catching up on schizophrenia: Natural history and neurobiology, Neuron 28 (2000), 325-334.

[21] M. W. Lin, P. Sham, H. G. Hwu, D. Collier, R. Murray, J. F. Powell, Suggestive evidence for linkage of schizophrenia to markers on chromosome 13 in caucasian but not oriental populations, Hum Genet 99 (1997), 417-420.

[22] D. Mayer, K. Molawi, L. Martinez-Sobrido, A. Ghanem, S. Thomas, S. Baginsky, J. Grossmann, A. Garcia-Sastre, M. Schwemmle, Identification of cellular interaction partners of the influenza virus ribonucleoprotein complex and polymerase complex using proteomic-based approaches, J Proteome Res 6 (2007), 672-682.

[23] M. Maziade, Y. C. Chagnon, M. A. Roy, A. Bureau, A. Fournier, C. Merette, Chromosome 13q13-q14 locus overlaps mood and psychotic disorders: The relevance for redefining phenotype, Eur J Hum Genet 17 (2009), 1034-1042.

[24] S. Nakielny, G. Dreyfuss, Transport of proteins and rnas in and out of the nucleus, Cell 99 (1999), 677-690.

[25] M. M. Nothen, V. Nieratschker, S. Cichon, M. Rietschel, New findings in the genetics of major psychoses, Dialogues Clin Neurosci 12 (2010), 85-93.

[26] J. Ott: Utility programs for analysis of genetic linkage, program, hwe version 1.10. New York, Columbia University, 1988 ,

[27] V. Peralta, M. J. Cuesta, Psychometric properties of the positive and negative syndrome scale (panss) in schizophrenia, Psychiatry Res 53 (1994), 31-40.

[28] G. Ponce, J. Hoenicka, M. A. Jimenez-Arriero, R. RodriguezJimenez, M. Aragues, N. Martin-Sune, E. Huertas, T. Palomo, Drd2 and ankk1 genotype in alcohol-dependent patients with psychopathic traits: Association and interaction study, $\mathrm{Br} \mathrm{J}$ Psychiatry 193 (2008), 121-125.

[29] S. M. Purcell, N. R. Wray, J. L. Stone, P. M. Visscher, M. C. O'Donovan, P. F. Sullivan, P. Sklar, Common polygenic variation contributes to risk of schizophrenia and bipolar disorder, Nature 460 (2009), 748-752.

[30] J. Shi, D. F. Levinson, J. Duan, A. R. Sanders, Y. Zheng, I. Pe'er, F. Dudbridge, P. A. Holmans, A. S. Whittemore, B. J. Mowry, A. Olincy, F. Amin, C. R. Cloninger, J. M. Silverman, N. G. Buccola, W. F. Byerley, D. W. Black, R. R. Crowe, J. R. Oksenberg, D. B. Mirel, K. S. Kendler, R. Freedman, P. V. Gejman, Common variants on chromosome 6p22.1 are associated with schizophrenia, Nature 460 (2009), 753-757.

[31] H. Stefansson, R. A. Ophoff, S. Steinberg, O. A. Andreassen, S. Cichon, D. Rujescu, T. Werge, O. P. Pietilainen, O. Mors, P. B. Mortensen, E. Sigurdsson, O. Gustafsson, M. Nyegaard, A. Tuulio-Henriksson, A. Ingason, T. Hansen, J. Suvisaari, J. Lonnqvist, T. Paunio, A. D. Borglum, A. Hartmann, A. FinkJensen, M. Nordentoft, D. Hougaard, B. Norgaard-Pedersen, Y. Bottcher, J. Olesen, R. Breuer, H. J. Moller, I. Giegling, H. B. Rasmussen, S. Timm, M. Mattheisen, I. Bitter, J. M. Rethelyi, B. B. Magnusdottir, T. Sigmundsson, P. Olason, G. Masson, J. R. Gulcher, M. Haraldsson, R. Fossdal, T. E. Thorgeirsson, U. Thorsteinsdottir, M. Ruggeri, S. Tosato, B. Franke, E. Strengman, L. A. Kiemeney, I. Melle, S. Djurovic, L. Abramova, V. Kaleda, J. Sanjuan, R. de Frutos, E. Bramon, E. Vassos, G. Fraser, U. Ettinger, M. Picchioni, N. Walker, T. Toulopoulou, A. C. Need, D. Ge, J. L. Yoon, K. V. Shianna, N. B. Freimer, R. M. Cantor, R. Murray, A. Kong, V. Golimbet, A. Carracedo, C. Arango, J. Costas, E. G. Jonsson, L. Terenius, I. Agartz, H. Petursson, M. M. Nothen, M. Rietschel, P. M. Matthews, P. Muglia, L. Peltonen, D. St Clair, D. B. Goldstein, 
K. Stefansson, D. A. Collier, Common variants conferring risk of schizophrenia, Nature 460 (2009), 744-747.

[32] S. Takeda, T. Fujiwara, F. Shimizu, A. Kawai, K. Shinomiya, S. Okuno, K. Ozaki, T. Katagiri, Y. Shimada, M. Nagata, T. Watanabe, A. Takaichi, Y. Kuga, M. Suzuki, H. Hishigaki, E. Takahashi, S. Shin, Y. Nakamura, Y. Hirai, Isolation and mapping of karyopherin alpha 3 (kpna3), a human gene that is highly homologous to genes encoding xenopus importin, yeast srp1 and human rch1, Cytogenet Cell Genet 76 (1997), 87-93.

[33] P. M. Visscher, M. E. Goddard, E. M. Derks, N. R. Wray, Evidence-based psychiatric genetics, aka the false dichotomy between common and rare variant hypotheses, Mol Psychiatry Epub ahead of print (2011).

[34] J. Voisey, C. D. Swagell, I. P. Hughes, C. P. Morris, A. van Daal, E. P. Noble, B. Kann, K. A. Heslop, R. M. Young, B. R. Lawford, The drd2 gene 957c $>$ t polymorphism is associated with posttraumatic stress disorder in war veterans, Depress Anxiety 26 (2009), 28-33.

[35] J. Voisey, C. D. Swagell, I. P. Hughes, J. P. Connor, B. R. Lawford, R. M. Young, C. P. Morris, A polymorphism in the dysbindin gene (dtnbp1) associated with multiple psychiatric disorders including schizophrenia, Behav Brain Funct 6 (2010), 41.
[36] J. Voisey, C. D. Swagell, I. P. Hughes, B. R. Lawford, R. M. Young, C. P. Morris, Analysis of hapmap tag-snps in dysbindin (dtnbp1) reveals evidence of consistent association with schizophrenia, Eur Psychiatry 25 (2010), 314-319.

[37] J. Wei, G. P. Hemmings, The kpna3 gene may be a susceptibility candidate for schizophrenia, Neurosci Res 52 (2005), 342-346.

[38] M. J. White, B. R. Lawford, C. P. Morris, R. M. Young, Interaction between $\mathrm{drd} 2 \mathrm{c} 957 \mathrm{t}$ polymorphism and an acute psychosocial stressor on reward-related behavioral impulsivity, Behav Genet 39 (2009), 285-295.

[39] N. R. Wray, S. M. Purcell, P. M. Visscher, Synthetic associations created by rare variants do not explain most gwas results, PLoS Biol 9 (2011), e1000579.

[40] H. Xu, C. B. Kellendonk, E. H. Simpson, J. G. Keilp, G. E. Bruder, H. J. Polan, E. R. Kandel, T. C. Gilliam, Drd2 c957t polymorphism interacts with the comt val158met polymorphism in human working memory ability, Schizophr Res 90 (2007), 104-107.

[41] H. Zhang, G. Ju, J. Wei, Y. Hu, L. Liu, Q. Xu, Y. Chen, Z. Sun, S. Liu, Y. Yu, Y. Guo, Y. Shen, A combined effect of the kpna3 and kpnb3 genes on susceptibility to schizophrenia, Neurosci Lett 402 (2006), 173-175. 


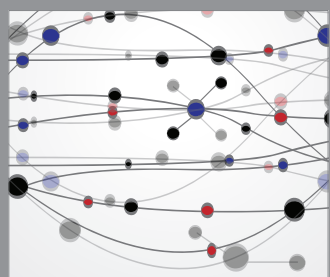

The Scientific World Journal
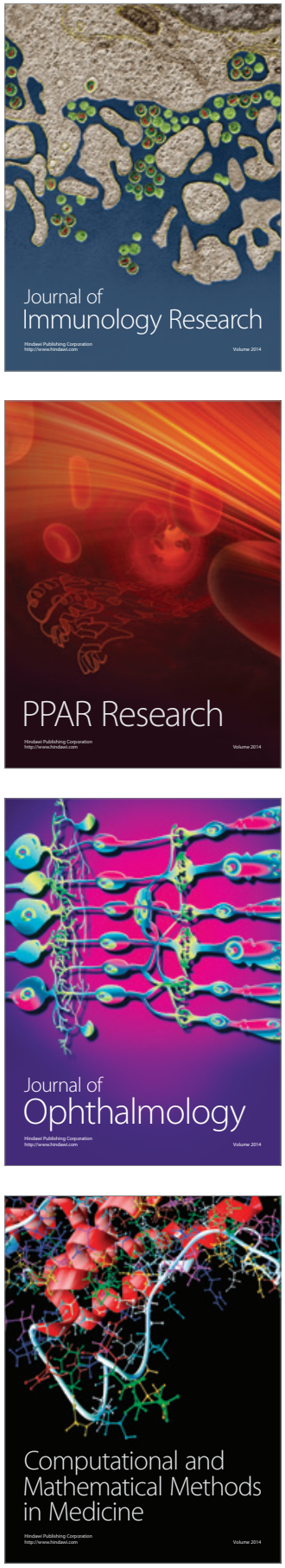

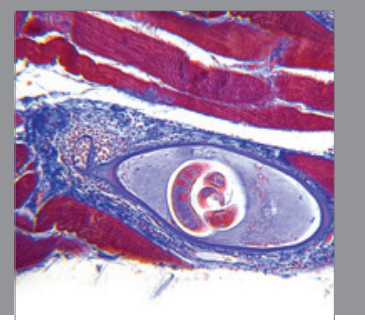

Gastroenterology

Research and Practice
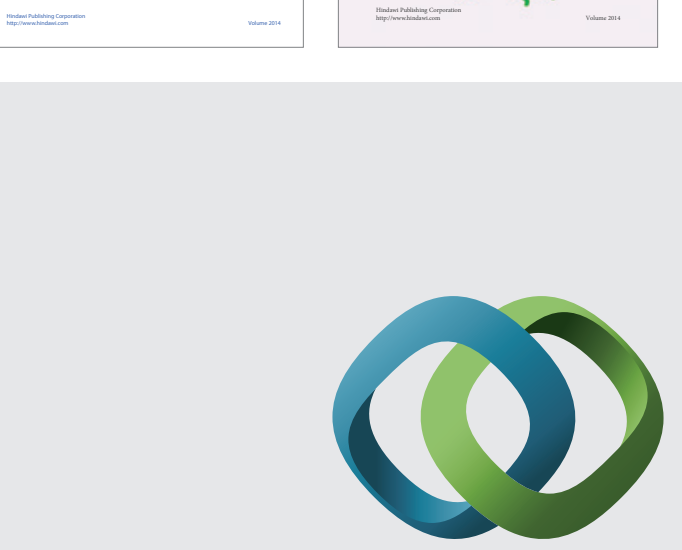

\section{Hindawi}

Submit your manuscripts at

http://www.hindawi.com
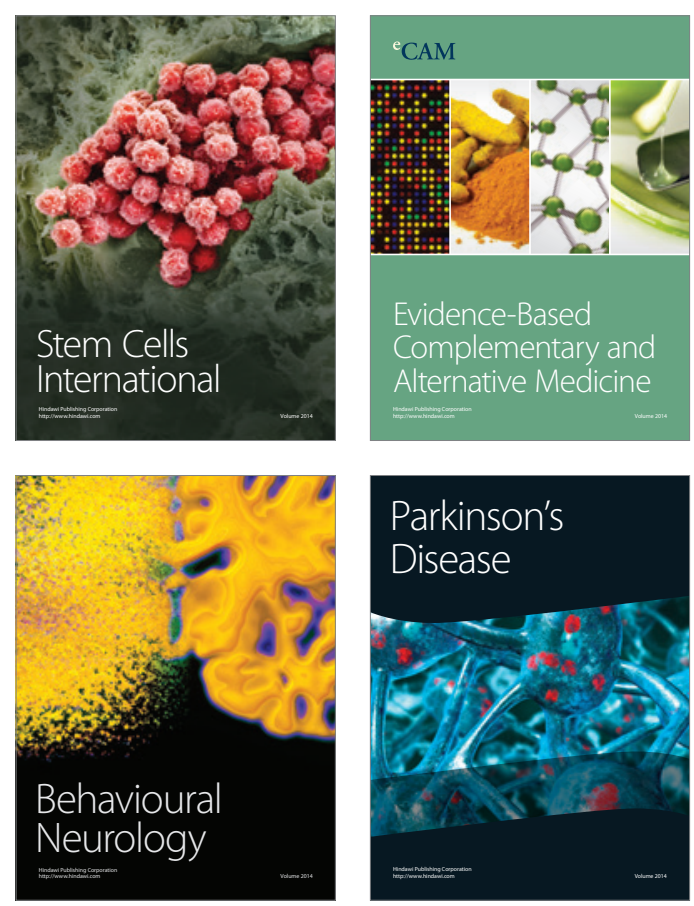

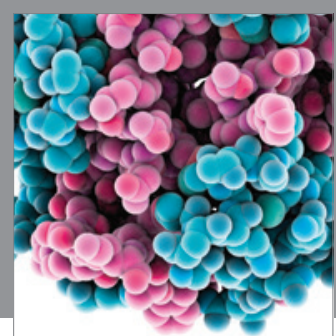

Journal of
Diabetes Research

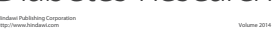

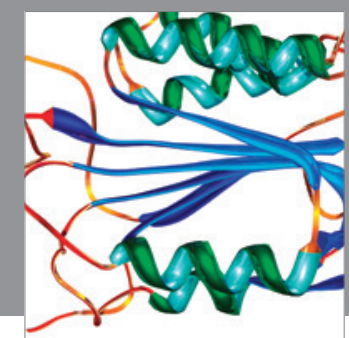

Disease Markers
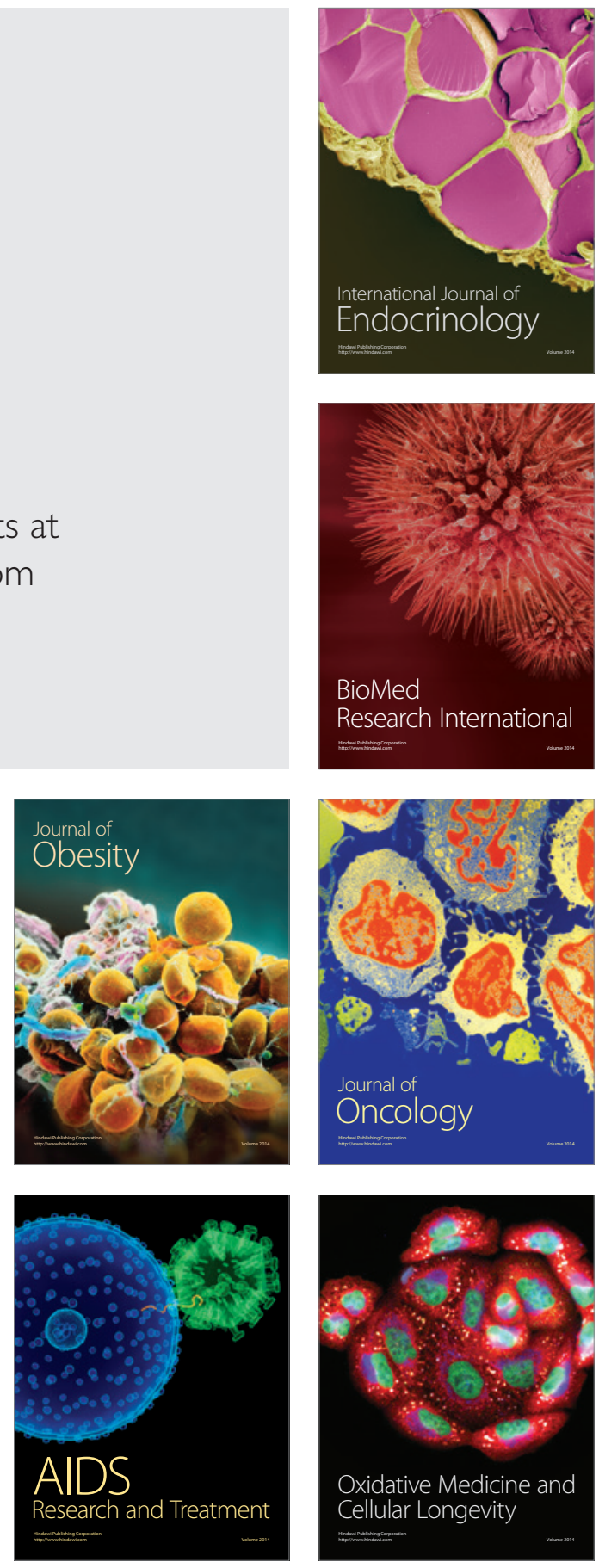